\title{
BMJ Open Coronary flow reserve during dobutamine stress in Takotsubo stress cardiomyopathy
}

\author{
Olov Collste, ${ }^{1}$ Per Tornvall, ${ }^{1}$ Mahbubul Alam, ${ }^{2}$ Mats Frick ${ }^{1}$
}

To cite: Collste 0 , Tornvall $P$, Alam M, et al. Coronary flow reserve during dobutamine stress in Takotsubo stress cardiomyopathy. BMJ Open 2015;5:e007671. doi:10.1136/bmjopen-2015007671

- Prepublication history for this paper is available online. To view these files please visit the journal online (http://dx.doi.org/10.1136/ bmjopen-2015-007671).

Received 15 January 2015 Revised 10 June 2015 Accepted 22 June 2015

\section{ABSTRACT}

Objectives: Takotsubo stress cardiomyopathy (TSC) is an increasingly recognised and diagnosed disease, although the underlying pathophysiology is still unknown. Our aim was to investigate the effect of the catecholamine dobutamine on coronary flow reserve (CFR) measured non-invasively in patients with TSC and controls. Our hypothesis was that dobutamine stress can induce microvascular dysfunction in patients with a previous episode of TSC.

Setting: This is a case-control study and a substudy of the Stockholm Myocardial Infarction with Normal Coronaries (SMINC) study. Elective dobutamine investigations were performed focusing on noninvasive measurements of CFR. The investigations were performed more than 6 months after the acute event.

Participants: 22 patients with a previous episode of TSC and 22 sex-matched and age-matched controls were recruited from the SMINC study. All patients with TSC had a previous normal cardiovascular MR investigation.

Results: CFR at low-dose dobutamine was significantly lower in the TSC group compared with controls, 1.51 and 1.72, respectively $(p=0.017)$. At high-dose dobutamine, CFR was 1.95 and 2.21 in the TSC group and controls, respectively ( $p=0.098)$.

Conclusions: We could not confirm that the catecholamine dobutamine induced microvascular dysfunction in patients with TSC. However, we found a small but significant difference in CFR at low-dose dobutamine, which implies that the role of microvascular function in TSC needs to be further explored.

\footnotetext{
${ }^{1}$ Department of Clinical Science and Education, Södersjukhuset, Karolinska Institutet, Stockholm, Sweden

${ }^{2}$ Department of Clinical Sciences, Danderyd Hospital, Karolinska Institutet, Stockholm, Sweden

Correspondence to Dr Olov Collste; olov.collste@sodersjukhuset. se
}

\section{INTRODUCTION}

In recent years, myocardial infarction with normal coronary arteries (MINCA) has been increasingly recognised and diagnosed. Owing to the frequent use of coronary angiography and new sensitive troponin assays in acute coronary syndromes, awareness of MINCA has increased. Recently, it was shown that MINCA occurs more frequently than previously thought, and our data indicate that the prevalence could be as high as $7-8 \%$

\section{Strengths and limitations of this study}

- Patients with Takotsubo stress cardiomyopathy (TSC) and controls were investigated with the catecholamine dobutamine.

- Non-invasive coronary flow reserve was assessed at low-dose and high-dose dobutamine.

- We could not confirm that the catecholamine dobutamine induced microvascular dysfunction in patients with TSC.

- We found a small but significant difference at low-dose dobutamine, which implies that the role of microvascular function in TSC needs to be further explored.

- One limitation is the relatively small sample size.

- A larger variation in means especially at highdose dobutamine might have obscured a true significant difference between patients with TSC and controls.

of all patients with acute coronary syndromes and approximately one-third of MINCA is constituted by Takotsubo stress cardiomyopathy (TSC). ${ }^{1}$ The aetiology of TSC is, to a large extent, unknown, although indications point towards a strong relationship between TSC and acute physiological or mental stress. ${ }^{2}$ Hence, there is a need to more fully explore the effects of stress in patients with a previous episode of TSC.

One possible explanation for TSC could be microvascular dysfunction. Previous studies have shown decreased coronary flow reserve (CFR) during the acute phase of TSG. ${ }^{34}$ CFR is thought to give an estimate of the maximal remaining flow reserve in a vessel, taking into account stenosis in the vessel, microvascular circulation and collateral flow. $^{5}$ Several studies have shown that CFR may be estimated by non-invasively recording the intracoronary flow velocity. ${ }^{5} 6$ Previously, the effects of dipyridamole, adenosine and cold pressor test on invasive and non-invasive CFR have been studied. ${ }^{48}$ However, dobutamine, a catecholamine that closely resembles epinephrine and thereby possibly more 
accurately mimics an acute TSC-like event, has not been studied regarding its effect on CFR in TSC.

In this study, we investigated the effects of the catecholamine dobutamine on non-invasive CFR in TSC. Our hypothesis was that CFR is reduced during dobutamine stress in patients with a previous episode of TSC.

\section{METHODS}

\section{Study group}

Twenty-two patients with a previous episode of TSC and 22 sex-matched and age-matched controls were recruited from the Stockholm Myocardial Infarction with Normal Coronaries study. The patients with TSC fulfilled the Mayo Clinic criteria for TSC. ${ }^{9}$ All patients with TSC were investigated with dobutamine stress tests focusing on non-invasive CFR, performed more than 6 months after the acute event. All patients with TSC were previously investigated with cardiovascular MR (CMR) and found to have no signs of previous myocardial infarction (myocardial necrosis) or myocarditis. The controls were sexmatched and age-matched volunteers recruited from the Stockholm metropolitan area. The controls were not investigated with coronary angiography prior to this study but had no signs or symptoms of coronary artery disease and a normal exercise stress test prior to inclusion into the study. Treatment with $\beta$-blockers was withheld on the day before examination. Current smokers were asked not to smoke on the day of examination.

\section{CFR}

All patients and controls were investigated before and during dobutamine stress. A Philips (Amsterdam, The Netherlands) iE33 was used to obtain echocardiograms. First the left ventricular (LV) ejection fraction (EF) and function of the mitral, tricuspid and aortic valve were recorded. For the flow velocity recordings a $3.5 \mathrm{MHz}$ high-frequency probe was used. The filter settings were kept at $150 \mathrm{~Hz}$ and gains were adjusted to the lowest possible level to minimise noise. A $10 \mathrm{~mm}$ sample volume was used. A Doppler velocity range of -15 to $15 \mathrm{~cm} / \mathrm{s}$ was selected but modified according to the maximum flow velocity achieved during increasing dobutamine levels. At rest, the flow velocity curve of the left anterior descending (LAD) artery was assessed from an apical two-chamber view (figure 1). If necessary, SonoVue (Bracco, Milano, Italy) contrast was used to enhance the flow velocity signal. The images obtained with Doppler were analysed using Syngo Dynamics software (Siemens Healthcare, Erlangen, Germany).

\section{Dobutamine stress}

The dobutamine stress examination was performed approximately 1 year and 8 months (mean 619 \pm 297 days) after the acute event for the TSC group and according to the consensus statement of the European Association of Echocardiography. ${ }^{10}$ An increasing dose of dobutamine was administered $(5,10,20,30$ and finally $40 \mu \mathrm{g} / \mathrm{kg} / \mathrm{min}$ ) and, if necessary, an additional dose of atropine was administered, until the heart rate reached $>85 \%$ of calculated maximum heart rate. The flow velocity curve in $\mathrm{LAD}$ was assessed at low dose $(10 \mu \mathrm{g} / \mathrm{kg} / \mathrm{min}$ dobutamine $)$ and at high dose $(40 \mu \mathrm{g} /$ $\mathrm{kg} / \mathrm{min}$ dobutamine).

Non-invasive CFR was calculated from the peak diastolic velocity in LAD at rest, low-dose and high-dose dobutamine, where CFR is the velocity during stress/velocity at rest. A mean of three consecutive cycles was used to calculate all parameters during dobutamine stress.

\section{Intraobserver and interobserver variability}

All the CFR data were analysed by an experienced echocardiographer, and analysed a second time by the same echocardiographer. An analysis was also performed by an independent echocardiographer not originally involved in the study. The three separate analyses were
Figure 1 Assessing the flow velocity curve in the left anterior descending artery (TSC,

Takotsubo stress cardiomyopathy).

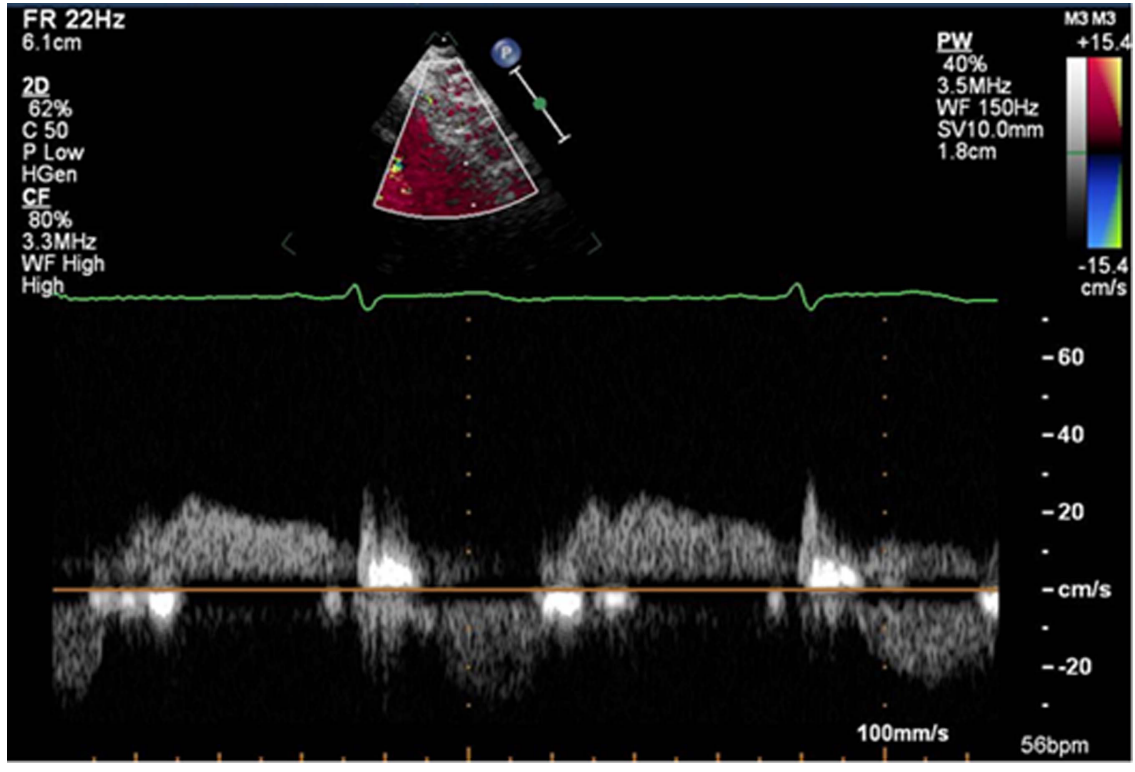


Table 1 Baseline characteristics

\begin{tabular}{llll}
\hline & Takotsubo & Control & p Value \\
\hline Number & 22 & 22 & NA \\
Age, years (mean) & 62.9 & 62.9 & NA \\
Gender (male/female) & $1 / 21$ & $1 / 21$ & NA \\
Present smoker (\%) & 18 & 0 & 0.04 \\
Diabetes mellitus (\%) & 4.5 & 0 & 0.33 \\
Hypertension (\%) & 45 & 23 & 0.12 \\
ß-Blocker (\%) & 50 & 14 & 0.01 \\
Calcium blocker (\%) & 18 & 0 & 0.04 \\
ACE-inhibitor (\%) & 45 & 14 & 0.02 \\
\hline NA, not applicable. & & &
\end{tabular}

combined for calculation of non-invasive CFR and intraclass correlation coefficient to estimate intraobserver and interobserver variability. The intraobserver variability was 0.46 and 0.78 for low-dose and high-dose dobutamine, respectively. Interobserver variability during dobutamine stress was 0.61 and 0.79 for low dose and high dose, respectively.

\section{STATISTICS}

The Student's t test with equal variances not assumed was used to calculate the mean values for the two independent groups and the $95 \%$ CI for the main outcome variables. By including 22 patients and 22 controls in the respective groups, the study had a power of $80 \%$ to detect a difference in CFR of $20 \%(p<0.05)$. Statistical analysis was made with SPSS V.22 (IBM, Armonk, New York, New York, USA). A p value of $<0.05$ was considered significant.

\section{RESULTS}

\section{Baseline characteristics}

The mean age of the TSC and control group at the time of the dobutamine stress investigation was 62.9 and 62.9 years, respectively. There were no significant differences in medical history of hypertension or diabetes mellitus between the groups. However, there were differences in current smoking and medications between the groups (table 1).

All patients and controls had normal systolic LV and right ventricular function with $\mathrm{LV}-\mathrm{EF}>55 \%$ as well as absence of regional LV or right ventricular hypokinesia. Both groups had normal LV and right ventricular dimensions (data not shown).

\begin{tabular}{llll} 
Table 2 & \multicolumn{2}{l}{ Coronary flow reserve $($ CFR) } \\
\hline & Takotsubo & Control & p Value \\
\hline CFR low & $1.51( \pm 0.23)$ & $1.72( \pm 0.33)$ & 0.017 \\
CFR high & $1.95( \pm 0.36)$ & $2.21( \pm 0.63)$ & 0.098 \\
\hline
\end{tabular}

Mean values $(95 \% \mathrm{Cl})$.

The Student $t$ test with equal variances not assumed was used to calculate the mean values for the two independent groups and to calculate the $95 \% \mathrm{Cl}$ for the main outcome variables.

\section{CFR}

All patients with TSC and controls reached $>85 \%$ of calculated maximum heart rate during dobutamine stress without the addition of atropine. Resting heart rate was similar for the patients with TSC and controls $(68 \mathrm{bpm}$ for both) as well as during low-dose dobutamine (99 vs $94 \mathrm{bpm}, \mathrm{p}=$ non-significant). Maximum heart rate at highdose dobutamine was also similar between the groups (131 vs $134 \mathrm{bpm}, \mathrm{p}=$ non-significant). CFR at low-dose dobutamine was significantly lower in patients with TSC compared with controls (1.51 and 1.72, $\mathrm{p}=0.017)$. Although the CFR was higher in controls during highdose dobutamine, the results did not reach the significance level (table 2 and figure 2).

\section{DISCUSSION}

In this, to the best of our knowledge, first study on microvascular function in TSC performed with a catecholamine, we could not confirm that dobutamine induced microvascular dysfunction. However, we did find a significant difference between patients with TSC and controls at low-dose dobutamine.

Although the effects of dobutamine stress on CFR in TSC have not been studied previously, there are several studies where the effects of adenosine or dipyridamole have been studied. In a study by Kume et al, the effect of adenosine on CFR in eight patients with TSC was invasively measured. They found a reduced CFR in the acute phase with normalisation after 3 weeks. ${ }^{11}$ Similar results have been shown by Meimoun et at using non-invasive CFR to measure the effect of adenosine during the acute TSC event and after 4 weeks. In contrast to these results, a study by Sganzerla et $a l^{12}$ of seven patients with

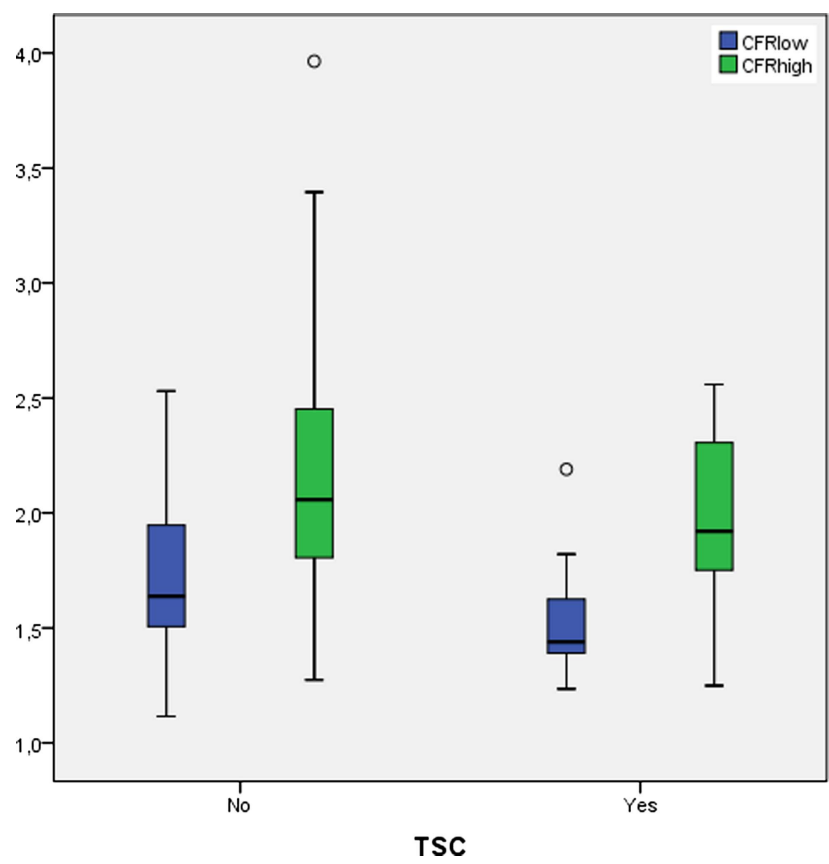

Figure 2 Results of coronary flow reserve. Thick horizontal line, median value; boxes, IQR and whiskers $95 \% \mathrm{Cl}$. 
TSC, showed no difference in non-invasive CFR using adenosine on admission, compared with its use after 3 weeks. However, patients with hypertension, diabetes mellitus and present smoking were all excluded from participating in the latter study. It should be noted that no control group was used in any of these studies. Rigo et $a l^{8}$ measured CFR non-invasively using dipyridamole. They compared 30 patients with TSC against 30 reasonably matched controls (mean 68 and 56 years, respectively). CFR in the acute phase was lower in the TSC group compared with the controls, while measurements on day 7 and after 6 months showed a normalisation of CFR. In summary, in most previous studies, CFR was reduced in the subacute phase in patients with TSC but normalised after a few weeks or months.

In contrast to CFR measured using adenosine, we found a small but significant difference using the catecholamine dobutamine more than 6 months after the acute event. Furthermore, there are obvious pharmacological differences between adenosine and dobutamine, where dobutamine is a catecholamine and in many ways is similar to physiologically active epinephrine and norepinephrine. Non-invasive CFR stimulated by dobutamine has not been compared with invasive CFR previously, but when compared to non-invasive CFR derived from adenosine, a good correlation has been shown. ${ }^{13}$ One plausible explanation for the lower CFR at low-dose dobutamine in the TSC group in our study could be microvascular dysfunction provoked by dobutamine. ${ }^{2}$ In a recent study by Patel $e t a l,{ }^{14}$ the authors concluded that coronary vascular reactivity is impaired in patients with TSC. This study was not carried out using a catecholamine; instead, acetylcholine was used to induce a coronary microvascular reaction. In a study by Barletta et $a l^{7}{ }^{7}$ a reduced CFR was shown with the cold pressor test 2 years after the acute event in 17 patients with TSC in comparison with seven controls. In contrast to our study, the authors used myocardial perfusion echocardiography to measure CFR. ${ }^{15}{ }^{16}$ In conclusion, there is evidence for normal CFR among patients with TSC when using vessel dilation to induce stress several months after the acute event. However, when using a cold pressor test or a catecholamine to induce stress, there is some evidence for reduced CFR (our study provides conflicting evidence). Furthermore, impaired coronary vascular reactivity has been shown more than 1 year after the acute event, also supporting microvascular dysfunction in TSC.

Using dobutamine stress at 1 year and 8 months after the acute event, we found that CFR was similar at highdose dobutamine but significantly lower at low-dose dobutamine in patients with TSC when compared with controls. There are three possible explanations for these conflicting results: (1) we induced a mild microvascular dysfunction in patients with TSC using dobutamine stress that is only significant compared with controls at low-dose dobutamine. (2) CFR was mildly pathological since the acute event. The differences between points
(1) and (2) would be that microvascular dysfunction is either induced during stress or chronically impaired. (3) Baseline characteristics are the explanation for pathological CFR during low-dose dobutamine stress. In all three instances, a more challenging assessment of peak coronary flow velocity could have influenced the results at high-dose dobutamine. In other words, a larger variation in means at high-dose dobutamine might have obscured a true significant difference between patients with TSC and controls. In conclusion, despite having a normal ventricular function at rest and during stress, patients with TSC exhibit a mildly reduced CFR, possibly because of differences in baseline characteristics or a combination of this and microvascular dysfunction induced during dobutamine stress.

In the present study, we achieved adequate levels of stress during the dobutamine infusion. All patients reached $>85 \%$ of calculated maximum heart rate at highdose dobutamine without using additional atropine. ${ }^{17}$ We chose not to withhold $\beta$-blocker treatment for more than 1 day before examination, for ethical reasons. Thus, one could expect $\beta$-blockers still influencing the results in patients with TSC and controls with $\beta$-blocker treatment. However, the supposedly negative effect of selective $\beta 1$-blockers such as metoprolol on CFR can be questioned. ${ }^{18}$ Hodgson et al invasively measured the effect of $\alpha$-blockers, non-selective $\beta$-blockers and selective $\beta 1$-blockers on coronary vascular resistance index and CFR and found that selective $\beta 1$-blockers had no effect on resting coronary velocity or coronary vascular resistance index. Moreover, in two other studies using adenosine and dipyridamole, respectively, pretreatment with the selective $\beta 1$-blocker metoprolol increased CFR. ${ }^{19} 20$

The effects of ACE inhibitors on CFR resemble the effects of selective $\beta 1$-blockers. Previous studies have shown that ACE inhibitors increase CFR. ${ }^{21-23}$ Thus, if metoprolol and ACE inhibitors increase CFR it would thereby result in a decreased difference between our study groups. In other words, the greater prevalence of patients treated with selective $\beta 1$-blocker and ACE inhibitor in our TSC group may have concealed greater differences between patients with TSC and controls.

One strength of this study is the selection of patients with TSC as well as the sex-matched and age-matched control group. The patients with TSC were investigated with coronary angiography, echocardiography and chest CT to exclude pulmonary embolism, and CMR to exclude myocarditis. CMR has not been used in most other studies of patients with TSC, whereby the TSC diagnosis, in reality, could be something different. ${ }^{1}$ Our control group was chosen not from emergency department clientele, but from the general population, often with some participants born on the same day as some of the patients with TSC. This provided a good match, not just in gender, but also in age.

In a study performed by Redfors $e t a l^{24}$ a TSC episode was induced in rats, using isoproterenol infusion. Regional myocardial perfusion was assessed using myocardial 
contrast echocardiography, with the finding that apical perfusion was not impaired in the early phase of the isoproterenol-induced TSC episode. This could support the conclusion that impairment of myocardial function can occur without myocardial ischaemia in the acute phase. However, in another study by Szardien et $a l^{25} 12$ patients with TSC were studied using ventricular biopsies from the apical region. They found that endomyocardial capillary density was reduced due to the expansion of the extracellular matrix. This could result in a reduced CFR, as reported in our study.

The particular regional appearance of TSC cannot be explained by the findings in our study. However, it was only possible to investigate CFR in LAD. Regional wall motion disturbance in TSC has to be the subject of future preclinical and clinical studies.

\section{Limitations}

This study had several limitations. First, it had a relatively small sample size. Thereby, we cannot rule out the possibility that differences in baseline characteristics other than age and sex, such as hypertension or current smoking, might have influenced our results.

Second, we chose to use dobutamine instead of, for example, epinephrine, for ethical and safety reasons. One possible explanation for the lack of significant results at high-dose dobutamine stress is the fact that dobutamine only has a weak $\beta 2$ effect. In animal studies, the negative inotropic effect at high catecholamine doses in a TSC event is mediated via the $\beta 2$ receptors. ${ }^{26} 27$ Therefore, dobutamine, despite being a catecholamine, could possibly not be enough to precipitate microvascular dysfunction even at high doses.

Third, we do not have data on CFR prior to the acute episode in the patients with TSC. This limits our ability to conclude whether a reduced CFR at low-dose dobutamine is a cause of TSC, induced by the acute episode, and/or a secondary effect to differences in baseline characteristics.

Finally, our results for intraobserver and interobserver variability demonstrate that there are caveats with this method that should be taken into consideration when evaluating this method and the results of this study. As already mentioned, a larger variation in means especially at high-dose dobutamine might have obscured a true significant difference between patients with TSC and controls.

\section{CONCLUSION}

We could not confirm that the catecholamine dobutamine induced microvascular dysfunction in patients with TSC. However, we found a small but significant difference in CFR at low-dose dobutamine, which implies that the role of microvascular function in TSC needs to be further explored.

Contributors OC, PT, MA and MF made substantial contributions to the conception and design of the work. OC was responsible for the acquisition, analysis and interpretation (PT, MA and MF) of data. OC, PT, MA and MF: drafting the work and revising it critically for important intellectual content. OC, PT, MA and MF: final approval of the version to be published.

Funding This study was supported by the Swedish Heart Lung Foundation (grant number 20110735).

Competing interests None declared.

Patient consent Obtained.

Ethics approval This study was performed in accordance with the Declaration of Helsinki and good clinical practice. The study was approved by the Regional Ethical Review Board in Stockholm. Written consent was acquired from the study participants in the main study but oral consent was acquired for this substudy. ${ }^{1}$ This consent procedure was approved by the Regional Ethical Review Board in Stockholm.

Provenance and peer review Not commissioned; externally peer reviewed.

Data sharing statement No additional data are available.

Open Access This is an Open Access article distributed in accordance with the Creative Commons Attribution Non Commercial (CC BY-NC 4.0) license, which permits others to distribute, remix, adapt, build upon this work noncommercially, and license their derivative works on different terms, provided the original work is properly cited and the use is non-commercial. See: http:// creativecommons.org/licenses/by-nc/4.0/

\section{REFERENCES}

1. Collste $\mathrm{O}$, Sorensson $\mathrm{P}$, Frick $\mathrm{M}$, et al. Myocardial infarction with normal coronary arteries is common and associated with normal findings on cardiovascular magnetic resonance imaging: results from the Stockholm Myocardial Infarction with Normal Coronaries study. J Intern Med 2013;273:189-96.

2. Pilgrim TM, Wyss TR. Takotsubo cardiomyopathy or transient left ventricular apical ballooning syndrome: a systematic review. Int $J$ Cardiol 2008;124:283-92.

3. Yanagi S, Nagae K, Yoshida K, et al. [Evaluation of coronary flow reserve using Doppler guide wire in patients with ampulla cardiomyopathy: three case reports]. J Cardiol 2002;39:305-12.

4. Meimoun $\mathrm{P}$, Malaquin D, Sayah S, et al. The coronary flow reserve is transiently impaired in tako-tsubo cardiomyopathy: a prospective study using serial Doppler transthoracic echocardiography. J Am Soc Echocardiogr 2008;21:72-7.

5. Hozumi T, Yoshida K, Akasaka T, et al. Noninvasive assessment of coronary flow velocity and coronary flow velocity reserve in the left anterior descending coronary artery by Doppler echocardiography: comparison with invasive technique. J Am Coll Cardiol 1998;32:1251-9.

6. Hozumi T, Yoshida K, Ogata Y, et al. Noninvasive assessment of significant left anterior descending coronary artery stenosis by coronary flow velocity reserve with transthoracic color Doppler echocardiography. Circulation 1998;97:1557-62.

7. Barletta G, Del Pace S, Boddi M, et al. Abnormal coronary reserve and left ventricular wall motion during cold pressor test in patients with previous left ventricular ballooning syndrome. Eur Heart $J$ 2009;30:3007-14

8. Rigo F, Sicari R, Citro R, et al. Diffuse, marked, reversible impairment in coronary microcirculation in stress cardiomyopathy a Doppler transthoracic echo study. Ann Med 2009;41:462-70.

9. Prasad A, Lerman A, Rihal CS. Apical ballooning syndrome (Tako-Tsubo or stress cardiomyopathy): a mimic of acute myocardial infarction. Am Heart J 2008;155:408-17.

10. Sicari R, Nihoyannopoulos P, Evangelista A, et al., European Association of Echocardiography. Stress echocardiography expert consensus statement: European Association of Echocardiography (EAE) (a registered branch of the ESC). Eur J Echocardiogr 2008;9:415-37.

11. Kume T, Akasaka T, Kawamoto T, et al. Assessment of coronary microcirculation in patients with takotsubo-like left ventricular dysfunction. Circ J 2005;69:934-9.

12. Sganzerla P, Alioto G, Funaro A, et al. Transthoracic Doppler ultrasound coronary flow reserve evaluation: preliminary insights into pathophysiology of Takotsubo cardiomyopathy. J Cardiovasc Med (Hagerstown) 2008;9:1229-34.

13. Meimoun P, Sayah S, Tcheuffa JC, et al. Transthoracic coronary flow velocity reserve assessment: comparison between adenosine and dobutamine. J Am Soc Echocardiogr 2006;19:1220-8. 
14. Patel SM, Lerman A, Lennon RJ, et al. Impaired coronary microvascular reactivity in women with apical ballooning syndrome (Takotsubo/stress cardiomyopathy). Eur Heart J Acute Cardiovasc Care 2013;2:147-52.

15. Abdelmoneim SS, Dhoble A, Bernier M, et al. Quantitative myocardial contrast echocardiography during pharmacological stress for diagnosis of coronary artery disease: a systematic review and meta-analysis of diagnostic accuracy studies. Eur J Echocardiogr 2009;10:813-25.

16. Mattoso AA, Kowatsch I, Tsutsui JM, et al. Prognostic value of qualitative and quantitative vasodilator stress myocardial perfusion echocardiography in patients with known or suspected coronary artery disease. J Am Soc Echocardiogr 2013;26:539-47.

17. Ironson G, Taylor CB, Boltwood M, et al. Effects of anger on left ventricular ejection fraction in coronary artery disease. Am J Cardiol 1992;70:281-5.

18. Hodgson JM, Cohen MD, Szentpetery $\mathrm{S}$, et al. Effects of regional alpha- and beta-blockade on resting and hyperemic coronary blood flow in conscious, unstressed humans. Circulation 1989;79:797-809.

19. Bottcher M, Czernin J, Sun K, et al. Effect of beta 1 adrenergic receptor blockade on myocardial blood flow and vasodilatory capacity. J Nucl Med 1997;38:442-6.

20. Billinger M, Seiler C, Fleisch $\mathrm{M}$, et al. Do beta-adrenergic blocking agents increase coronary flow reserve? J Am Coll Cardiol 2001;38:1866-71.

21. Motz W, Strauer BE. Improvement of coronary flow reserve after long-term therapy with enalapril. Hypertension 1996;27:1031-8.
22. Kawata T, Daimon M, Hasegawa R, et al. Effect on coronary flow velocity reserve in patients with type 2 diabetes mellitus: comparison between angiotensin-converting enzyme inhibitor and angiotensin II type 1 receptor antagonist. Am Heart J 2006;151:798 e9-15.

23. Pauly DF, Johnson BD, Anderson RD, et al. In women with symptoms of cardiac ischemia, nonobstructive coronary arteries, and microvascular dysfunction, angiotensin-converting enzyme inhibition is associated with improved microvascular function: a double-blind randomized study from the National Heart, Lung and Blood Institute Women's Ischemia Syndrome Evaluation (WISE). Am Heart $J$ 2011;162:678-84.

24. Redfors B, Shao Y, Wikstrom J, et al. Contrast echocardiography reveals apparently normal coronary perfusion in a rat model of stress-induced (Takotsubo) cardiomyopathy. Eur Heart J Cardiovasc Imaging 2014;15:152-7.

25. Szardien S, Mollmann H, Willmer M, et al. Molecular basis of disturbed extracellular matrix homeostasis in stress cardiomyopathy. Int J Cardiol 2013;168:1685-8.

26. Lyon AR, Rees PS, Prasad S, et al. Stress (Takotsubo) cardiomyopathy - a novel pathophysiological hypothesis to explain catecholamine-induced acute myocardial stunning. Nat Clin Pract Cardiovasc Med 2008;5:22-9.

27. Shao $\mathrm{Y}$, Redfors $\mathrm{B}$, Scharin Tang $\mathrm{M}$, et al. Novel rat model reveals important roles of beta-adrenoreceptors in stress-induced cardiomyopathy. Int J Cardiol 2013;168:1943-50. 